\title{
DETECTION OF POLARIZED QUASI-PERIODIC MICROSTRUCTURE EMISSION IN MILLISECOND PULSARS
}

\author{
Kishalay De ${ }^{1,3}$, Yashwant Gupta ${ }^{2}$, and Prateek Sharma ${ }^{1}$ \\ ${ }^{1}$ Department of Physics, Indian Institute of Science, Bangalore 560012, India; kde@caltech.edu \\ ${ }^{2}$ National Centre for Radio Astrophysics, TIFR, Pune University Campus, Post Bag 3, Pune 411007, India \\ Received 2016 October 16; revised 2016 November 14; accepted 2016 November 22; published 2016 December 5
}

\begin{abstract}
Microstructure emission, involving short timescale, often quasi-periodic, intensity fluctuations in subpulse emission, is well known in normal period pulsars. In this Letter, we present the first detections of quasi-periodic microstructure emission from millisecond pulsars (MSPs), from Giant Metrewave Radio Telescope observations of two MSPs at 325 and $610 \mathrm{MHz}$. Similar to the characteristics of microstructure observed in normal period pulsars, we find that these features are often highly polarized and exhibit quasi-periodic behavior on top of broader subpulse emission, with periods of the order of a few $\mu$ s. By measuring their widths and periodicities from single pulse intensity profiles and their autocorrelation functions, we extend the microstructure timescale-rotation period relationship by more than an order of magnitude down to rotation periods $\sim 5 \mathrm{~ms}$, and find it to be consistent with the relationship derived earlier for normal pulsars. The similarity of behavior is remarkable, given the significantly different physical properties of MSPs and normal period pulsars, and rules out several previous speculations about the possible different characteristics of microstructure in MSP radio emission. We discuss the possible reasons for the non-detection of these features in previous high time resolution MSP studies along with the physical implications of our results, both in terms of a geometric beam sweeping model and temporal modulation model for micropulse production.
\end{abstract}

Key words: methods: observational - pulsars: general - pulsars: individual (J0437-4715, J2145-0750) techniques: polarimetric

\section{INTRODUCTION}

Millisecond pulsars (MSPs), having rotation periods ranging from about one millisecond to few tens of milliseconds, have contributed significantly to our understanding of stellar evolution and the pulsar radio emission process. Believed to arise as the end products of mass accretion from a companion, the high rotation frequencies of these objects imply that the corotating magnetosphere (and hence the radio emission region) is significantly smaller in these sources compared to normal period pulsars. Nonetheless, previous studies have shown that although MSPs have drastically different physical parameters as compared to those of normal period pulsars, there are striking similarities in their radio emission properties as well as interesting differences (e.g., Kramer et al. 1998, 1999a; Sallmen 1998).

Single pulses in normal period pulsars are known to exhibit a rich variety of short timescale variations. In particular, microstructure emission has been long known to be a common feature of single pulse emission (first detected by Craft et al. 1968) and typically involves intensity variations at milli-period timescales, corresponding to timescales of a few hundred microseconds for normal period pulsars (e.g., Hankins 1972). Previous studies have shown that these features have typical widths for a given pulsar and often appear as a train of quasi-periodic pulses, with their timescales showing a strong correlation with the pulsar period (e.g., Kramer et al. 2002; Mitra et al. 2015).

The characterization of microstructure emission in MSPs still remains as one of the elusive aspects of the field. The single pulse properties (excluding giant radio pulses) of only four

\footnotetext{
3 Present address: California Institute of Technology, 1200 E. California Boulevard, Pasadena, CA 91125, USA.
}

MSPs have been explored to date-PSR J0437-4715 (Jenet et al. 1998), PSR B1534+12 (Sallmen 1998), PSR J1713 +0747 (Liu et al. 2016), and PSR B1937+21 (Jenet et al. 2001). Jenet et al. (1998) did one of the highest time resolution single pulse studies ever done on MSPs, observing the MSP J0437-4715 at L band, and reported that there was no evidence of microstructure down to a time resolution of $80 \mathrm{~ns}$. Similar reports were provided by Sallmen (1998) for B1937 +21 and B1534+12 and by Liu et al. (2016) for J1713+0747, claiming the absence of microstructure down to sub-microsecond time resolutions. However, the very limited number of such studies, particularly with high time resolution and sensitivity at low frequencies, leave a significant parameter space of this field unexplored.

Observations of sub-structures in MSP single pulses is challenging for a good number of reasons. On one hand, they are known to be relatively less luminous compared to their slower counterparts, and on the other, they require significantly higher time resolutions to study. While observations at low frequencies are favorable owing to the steep spectra of these sources, the effects of the interstellar medium, unless removed, pose a challenge to very high time resolution studies. Hence, in order to do such studies, one requires a telescope with very high sensitivity to be capable of detecting single pulses with good signal-to-noise ratios (S/Ns), as well as an appropriate backend capable of mitigating the dispersive effects of the interstellar medium with coherent dedispersion over reasonably large bandwidths. The Giant Metrewave Radio Telescope (GMRT) provides a unique platform for revisiting some important unanswered questions on the single pulse properties of MSPs, capitalizing on its high sensitivity at meter wavelengths and the recent development of a real-time coherent dedispersion system (De \& Gupta 2016). In this 
Table 1

Observing Parameters for the Pulsar Observations Carried Out in This Campaign, Along with Previously Known Parameters of the Respective Pulsars

\begin{tabular}{|c|c|c|c|c|c|c|c|c|c|c|c|}
\hline Pulsar Name & $\begin{array}{l}\text { Frequency Band } \\
\qquad(\mathrm{MHz})\end{array}$ & $\begin{array}{l}\text { Bandwidth } \\
\text { (MHz) }\end{array}$ & $\begin{array}{l}\text { Duration } \\
\text { (s) }\end{array}$ & NP & $\begin{array}{l}\text { Resolution } \\
(\mu \mathrm{s})\left(^{\circ}\right)\end{array}$ & MJD & $\begin{array}{l}\text { Period } \\
(\mathrm{ms})\end{array}$ & $\begin{array}{c}\mathrm{DM} \\
(\mathrm{pc} / \mathrm{cc})\end{array}$ & $\begin{array}{l}\text { S400 } \\
(\mathrm{mJy})\end{array}$ & $\begin{array}{c}\mathrm{RM} \\
\left(\operatorname{rad~m}^{-2}\right)\end{array}$ & $\begin{array}{c}\tau_{\text {scatt }} \\
(\mu \mathrm{s})\end{array}$ \\
\hline J0437-4715 & 610 & 32 & 1500 & 260534 & $0.96(0.06)$ & 57367 & 5.7574 & 2.6448 & 550 & 0.0 & 0.004 \\
\hline $\mathrm{J} 2145-0750$ & 610 & 32 & 1920 & 119608 & $3.84(0.08)$ & 57367 & 16.0524 & 8.9976 & 100 & -1.3 & 0.031 \\
\hline J0437-4715 & 325 & 32 & 1200 & 208427 & $0.96(0.06)$ & 57380 & 5.7574 & 2.6448 & 550 & 0.0 & 0.047 \\
\hline $\mathrm{J} 2145-0750$ & 325 & 32 & 1500 & 93444 & $3.84(0.08)$ & 57381 & 16.0524 & 8.9976 & 100 & -1.3 & 0.351 \\
\hline J0437-4715 & 610 & 32 & 900 & 156320 & $0.96(0.06)$ & 57389 & 5.7574 & 2.6448 & 550 & 0.0 & 0.004 \\
\hline
\end{tabular}

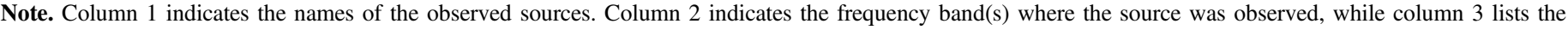

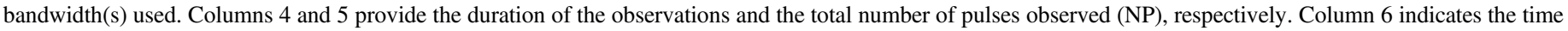

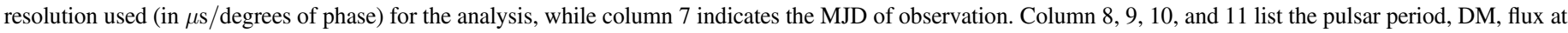

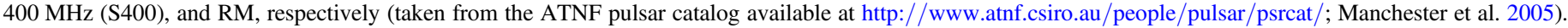
Column 12 indicates the expected scattering timescale for the pulsar at the given frequency band (derived from Cordes \& Lazio 2002).

Letter, we present results from a study of the full polarization properties of single pulses in two MSPs, concentrating on the characteristics of microstructure emission and its timescales.

\section{OBSERVATIONS}

The GMRT is a multi-element aperture synthesis telescope, consisting of 30 antennas, each with a diameter of $45 \mathrm{~m}$, separated by a maximum baseline of $25 \mathrm{~km}$ (Swarup et al. 1997). It can also be used for studies of compact objects like pulsars, by adding the signals from the antennas in an array mode (Gupta et al. 2000). We present observations of two MSPs (PSR J0437-4715 and PSR J2145-0750) at $325 \mathrm{MHz}$ and $610 \mathrm{MHz}$, selected based on their cataloged fluxes at $400 \mathrm{MHz}$ (S400 $>=100 \mathrm{mJy}$ ) and dispersion measures (DM $<=20 \mathrm{pc} /$ cc). As we expect to see microstructure emission at timescales of $0.001 \mathrm{P}$, the criterion for the selection of frequency bands was such that the expected scattering timescale at each frequency band (see Table 1) was smaller than the expected microstructure timescales. Wherever possible, we divided our observing time into multiple epochs to capitalize on serendipitous increase in the pulsar flux density due to refractive scintillation.

The observations were carried out using the Phased Array (PA) voltage beam mode (typically combining 14-16 antennas) of the GMRT Software Backend (Roy et al. 2010), which records the full Nyquist time resolution dual polarization voltage spectra for a given bandwidth, from a single PA beam of the telescope. The recorded voltages were subsequently coherently dedispersed using an offline version of the recently developed real-time coherent dedispersion pipeline (De \& Gupta 2016). The coherently dedispersed voltages (for two circular polarizations) were used to construct Stokes spectra with the desired time resolution (see Table 1). The time resolution used was optimized to detect sub-structures in single pulses with enough $\mathrm{S} / \mathrm{N}$, as well as to have similar phase resolutions for the two pulsars. Frequency-dependent instrumental gains were corrected using the time-averaged spectra for each polarization signal, while frequency-dependent phases due to instrumental delays and Faraday rotation were corrected by fitting the linear polarization angle of the average profile as a function of frequency across the band. The specific details of the observing parameters used for each epoch, along with the known parameters of the pulsars, are given in Table 1.

\section{DATA ANALYSIS}

The baselines for the individual Stokes parameters $I, Q, U$, and $V$ were adjusted to zero mean for all the data sets. We use the symbol $L=\sqrt{Q^{2}+U^{2}}$ to represent the total linear polarized intensity, and PA $=\frac{1}{2} \arctan \frac{U}{Q}$ to represent the angle of the linear polarization. For the individual folded profiles, we plot PA only if $L$ exceeds three times the baseline root mean square (rms) noise. For PSR J2145-0750, we did not detect a significant number of single pulses with high $\mathrm{S} / \mathrm{N}$ (peak $>10$ baseline rms) in our $325 \mathrm{MHz}$ data, and hence we do not include this data in our subsequent analysis. The folded profiles from our observations at each frequency band are given in Figures 1 and 2, for the pulsars J0437-4715 (at $325 \mathrm{MHz}$ and $610 \mathrm{MHz}$ ) and $\mathrm{J} 2145-0750$ (at $610 \mathrm{MHz}$ ), respectively, which are consistent with previously published polarization profiles at these frequencies (Navarro et al. 1997; Stairs et al. 1999).

\subsection{Autocorrelation Function (ACF)}

The timescales of microstructure emission, i.e., their widths and periodicity (if present) are of fundamental importance in microstructure studies. The ACF is a useful tool to measure these timescales in individual pulses, as well as to detect the presence of "preferred" timescales (if any) in a large ensemble of pulses. We use the definition of the ACF given in Lange et al. (1998) for our analysis. In normal period pulsars, the ACFs of individual subpulses are primarily dominated by a smooth bell-shaped curve centered at zero lag, with micropulses forming weak (or occasionally strong) modulations on the broader decline of the ACF. In cases where quasi-periodic microstructure is present, they form periodic maxima in the $\mathrm{ACF}$, with the time lag of the first peak representing the characteristic separations of the micropulses (e.g., Cordes et al. 1990).

\subsection{Searching for Periodic Microstructure}

We searched for the presence of microstructure in our data set by browsing through hundreds of single pulses from each observation. While we did attempt to find the presence of microstructure numerically, as done previously using ACF slope-flattening/maxima searches (e.g., Lange et al. 1998), we found that the diverse morphology of single pulses, along with the lack of a large number of single pulses with very high $\mathrm{S} / \mathrm{N}$ made such automated techniques difficult to implement accurately. Hence, for each pulsar (at each frequency band), 


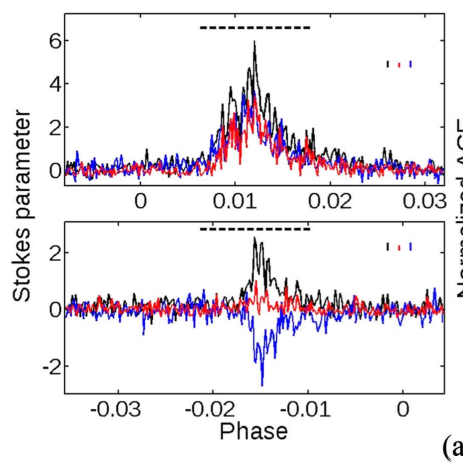

(a)

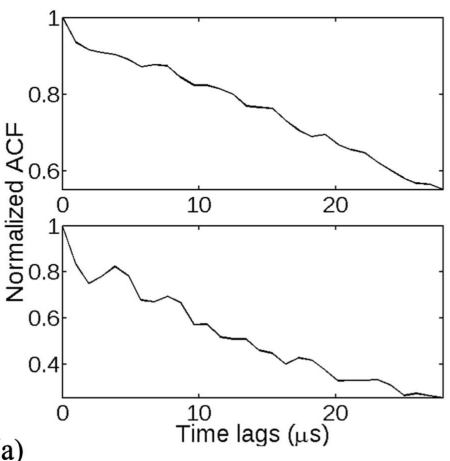

Full Stokes Profile for J0437-4715 at $325 \mathrm{MHz}$
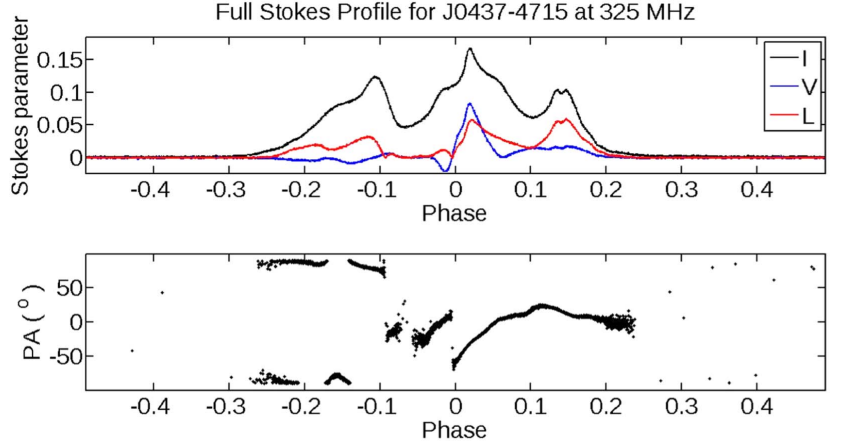

(c)
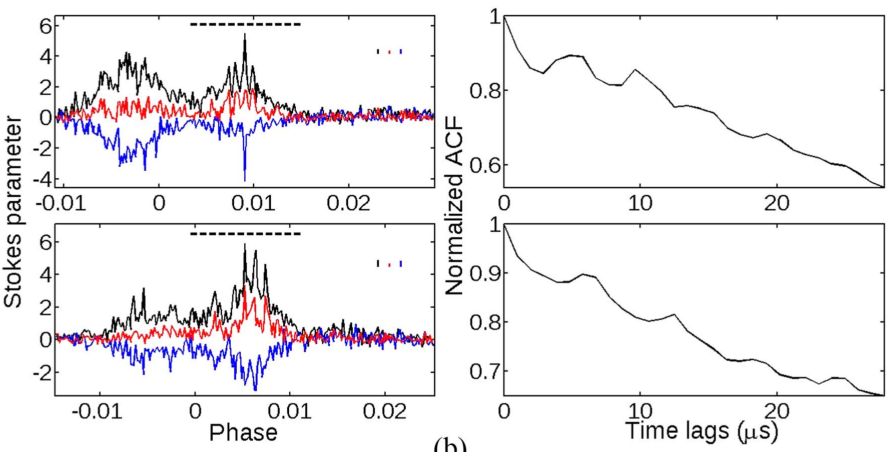

(b)

Full Stokes Profile for J0437-4715 at $610 \mathrm{MHz}$
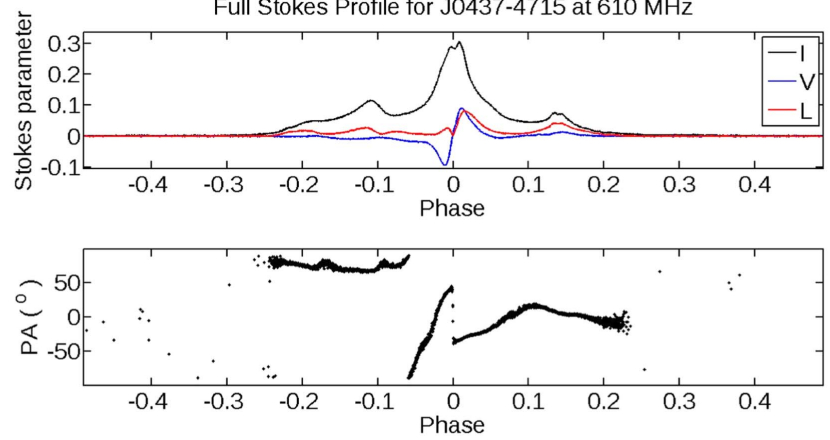

(d)

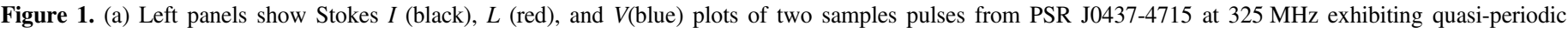

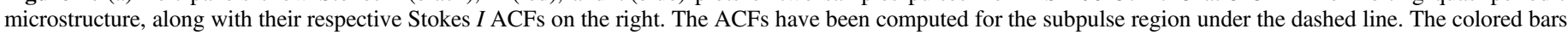

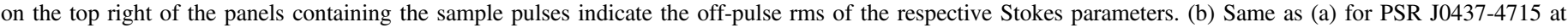

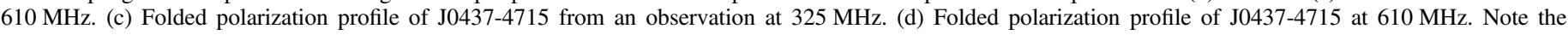
prominent oscillations in the single pulse ACFs produced by quasi-periodic microstructure.

we manually browsed through the brightest single pulses (with a peak $\mathrm{S} / \mathrm{N}$ threshold above a certain value) closely examining their morphology and ACFs. When periodic micropulses were found by visual inspection of the single pulse time series, we cross-checked the ACF for the presence of periodic maxima consistent with the separations and widths of the time domain features, and if present, recorded the first maxima in the ACF as the periodicity of the micropulses. Since both pulsars in our sample have low DMs, we also examined the off-pulse region around pulses that show microstructure, along with the corresponding zero DM time series, to exclude the possibility of RFI features mimicking microstructure, and did not find evidence for such contamination. Unless mentioned otherwise, we refer only to timescales of Stokes $I$ in subsequent discussion.

\section{RESULTS}

\subsection{PSR J0437-4715}

We found a large number of single pulses with high $\mathrm{S} / \mathrm{N}$ (with threshold set at peak $>15$ baseline rms) from this source - detecting $>100$ pulses at $325 \mathrm{MHz}$ and $>800$ pulses at $610 \mathrm{MHz}$. The brightest single pulses are often highly polarized narrow spikes of emission, with widths $<10 \mu$ s and no substructure apparent down to our time resolutions, consistent with previous reports by Jenet et al. (1998). Manually browsing these pulses, we found a significant number $(\sim 10 \%)$ of pulses exhibiting microstructure at $610 \mathrm{MHz}$ (which are not necessarily the brightest pulses in the data set). We further detected microstructure in a few bright pulses at $325 \mathrm{MHz}$, although the number of such pulses was lower due to the relatively lower
$\mathrm{S} / \mathrm{N}$ of the data. A few such sample pulses from this source, exhibiting polarized quasi-periodic microstructure are shown in Figure 1.

Micropulses, when present, are typically highly polarized and appear either in the form of a train of three or more quasiperiodic modulations, or in the form of single pairs of micropulses. The modulations appear in Stokes $I, L$, and $V$ and are typically weak compared to the underlying subpulse emission. As most micropulses appear in the form of close unresolved trains, it was not possible to measure their widths from the single pulse ACFs. Hence, we recorded only the micropulse periodicities from the ACFs and show their distribution in Figure 3, along with the $\mathrm{S} / \mathrm{N}$ distribution of all pulses and pulses that exhibit microstructure. We note, however, that when micropulses are resolved, their widths are typically $\sim 2 \mu \mathrm{s}$, and hence unresolved with typical periodicities of $\sim 4 \mu$ s. The smaller number of bright pulses exhibiting microstructure at $325 \mathrm{MHz}$ did not allow us to construct meaningful distributions. Nonetheless, we report a median periodicity of $4.72 \mu \mathrm{s}$ found in quasi-periodic microstructure for this source at $325 \mathrm{MHz}$.

\subsection{PSR J2145-0750}

We did not detect a significant number of single pulses with high $\mathrm{S} / \mathrm{N}$ (peak $>10$ baseline rms) in any of the two observing epochs for this source at $325 \mathrm{MHz}$. However, in the single observing epoch at $610 \mathrm{MHz}$, we found $>700$ single pulses with peak $>15$ baseline rms. Browsing these single pulses, we found a good fraction $(\sim 15 \%)$ of the pulses to exhibit polarized quasi-periodic microstructure. Similar to J0437-4715, the brightest pulses are devoid of microstructure and exhibit a 

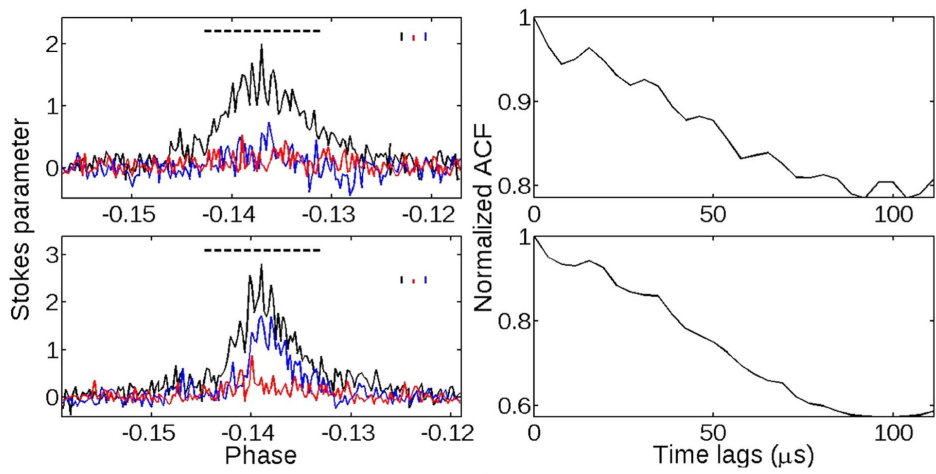

(a)
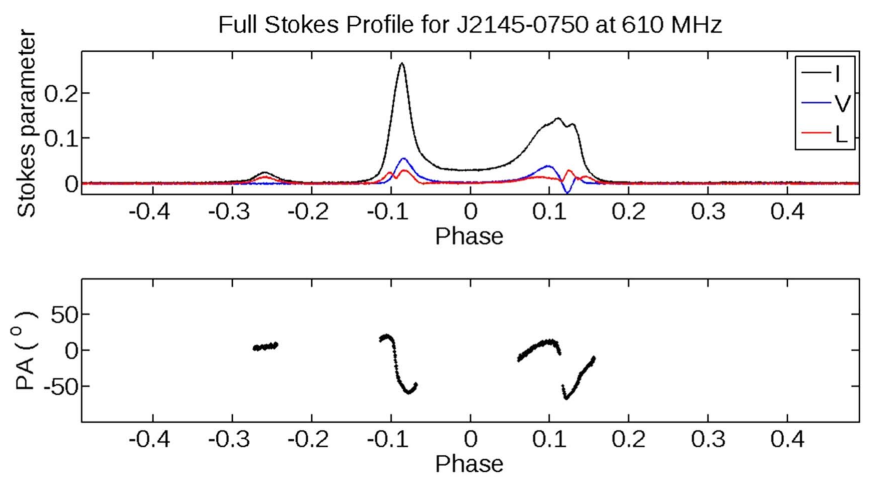

(b)

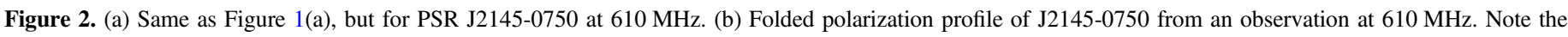
prominent periodic oscillations in the single pulse ACFs produced by periodic microstructure.

broad emission component only, while the relatively fainter pulses exhibiting microstructure consist of a broad subpulse component with weak modulations. A few typical pulses exhibiting quasi-periodic microstructure are shown in Figure 2.

We find micropulses to be highly polarized in this source, occasionally showing very high levels of circular polarization (see, for example, the lower panel in Figure 2(a)). Resolved micropulses are virtually absent in this source, as they always appear in quasi-periodic trains of four or more micropulses. As a result, we recorded only the periodicities of the microstructure from the single pulse ACFs and show their distribution in Figure 3, along with the distribution of $\mathrm{S} / \mathrm{N}$, as in the case of J0437-4715. Nonetheless, the microstructure periodicities suggest that the individual micropulse widths are $\gtrsim 8 \mu \mathrm{s}$.

\section{DISCUSSION}

The origin of microstructure emission remains as one of the most perplexing unanswered questions in our understanding of pulsar radio emission even though it is well established that microstructure is indeed an integral component of the emission process. In this Letter, we have reported on the first detections of microstructure emission in MSPs, which suggests that despite of the significant differences in the physical parameters of their magnetospheres, microstructure remains as one of the fundamental properties of radio emission in these sources. Indeed, the observation that the pulses exhibiting microstructure are typically not the brightest pulses in our data set suggests that there is likely an ensemble of weaker pulses below our detection threshold that exhibit microstructure, thereby indicating that it may be a fairly common occurrence in MSPs.

It is instructive to reflect upon the reasons for non-detection of these features in previous high time resolution studies of MSPs. First, it is important to note that the majority of previous single pulse studies of MSPs have had very limited sensitivity toward detection of single pulses and have concentrated on statistics of accumulated pulses over many periods (e.g., Jenet et al. 2001). Our study is one of the first reports of high sensitivity, high time resolution observations at relatively low frequencies (at and below $600 \mathrm{MHz}$ ), which forms an ideal combination for detection of micropulses from MSPs. Interestingly, Jenet et al. (1998) did a study on J0437-4715 with similar sensitivities at $1.4 \mathrm{GHz}$ and claimed that there was no evidence for microstructure emission. However, we note that their analysis concentrated on a search for microstructure features in the average ACF of single pulses, which often does not show the expected "breaks" when microstructure is weak or does not have a preferred timescale (Cordes et al. 1990). More recently, Liu et al. (2016) reported on the non-detection of microstructure emission in PSR $\mathrm{J} 1713+0747$ at L band, by analyzing the properties of the averaged ACF as well as single pulse ACFs of the brightest pulses. We note that higher frequency non-detections may also be due to a steeper spectrum of micropulses compared to subpulse emission (as shown for B2016 +28 by Cordes et al. 1990).

We now use our results to examine the microstructure quasiperiodicity-pulsar rotation period relationship down to the millisecond rotation periods, using the median quasi-periodicity of the distributions (Figure 3) as the typical periodicity and their median absolute deviation as the characteristic scatter. Since we find micropulses to be mostly unresolved from the underlying quasi-periodicity, it is difficult to estimate their intrinsic widths, and hence we do not discuss the micropulse width-rotation period relationship. We use previously reported microstructure periodicities in Kramer et al. (2002, Table 2) and Mitra et al. (2015, Table 3) along with our new results to extend the relationship. Note that previous reports combine observations from multiple frequencies of observation, and in cases where multiple reports were present (at different frequencies, different analysis techniques, or for different subpulse components), we only used the results with reported uncertainty estimates and averaged their timescales for our analysis. While there is some weak evidence for change in microstructure timescales across frequencies, the dependence is known to be very weak (e.g., Hankins et al. 1976; Ferguson \& Seiradakis 1978; Kramer et al. 2002). Hence, we do not expect such averaging to change the properties of the relationship significantly. As shown in Figure 4, we derive a relationship of

$$
P_{\mu}=(1.06 \pm 0.62) P_{r}^{(0.96 \pm 0.09)},
$$

where $P_{\mu}$ is the typical microstructure periodicity in units of $\mu \mathrm{s}$ and $P_{r}$ is the rotation period in units of ms. The uncertainties shown are at the $95 \%$ confidence level. The relationship is consistent with the linear relationships derived by Cordes (1979) and Mitra et al. (2015) from data for only normal period pulsars. This result indicates that there is a striking similarity of the properties of microstructure emission in normal and recycled pulsars. 
Histogram of peak S/N for J0437-4715 at $610 \mathrm{MHz}$

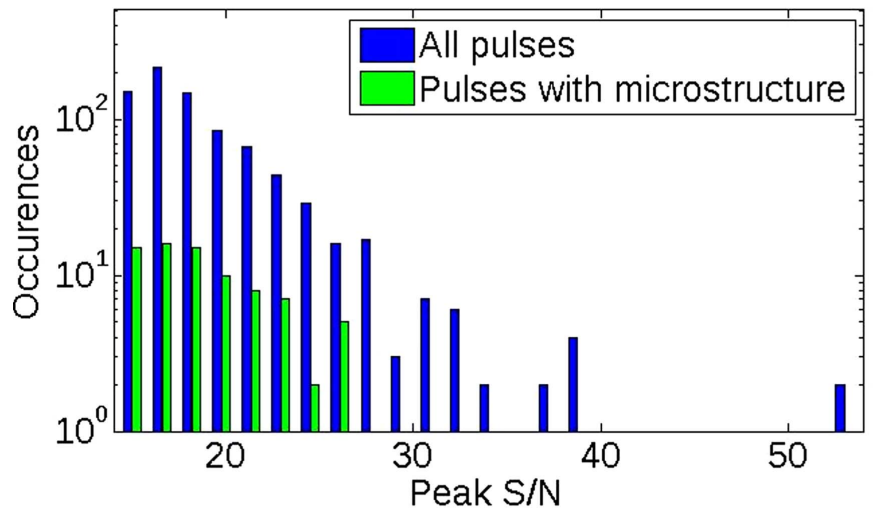

(a)

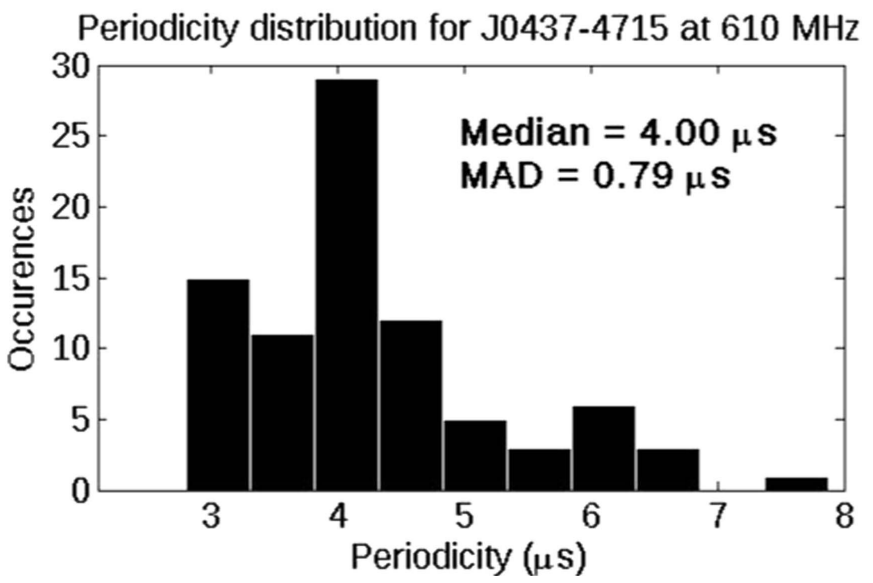

(c)
Histogram of peak S/N for J2145-0750 at $610 \mathrm{MHz}$

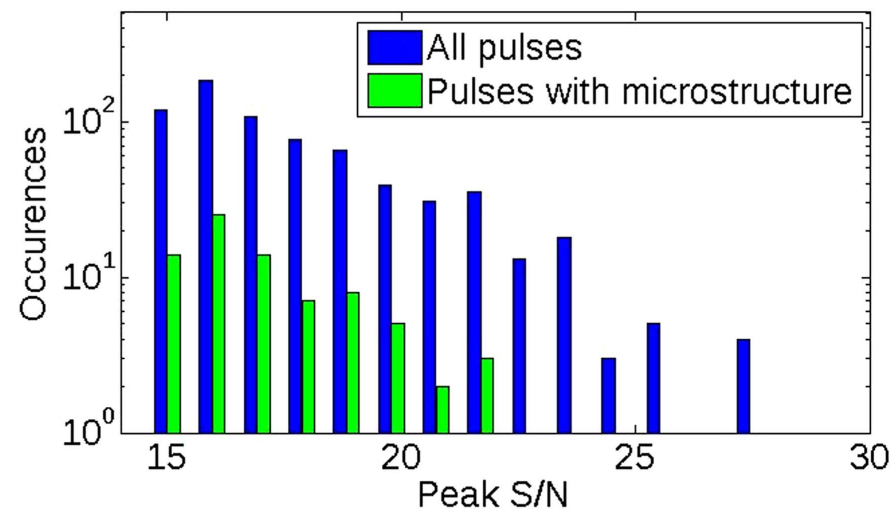

(b)

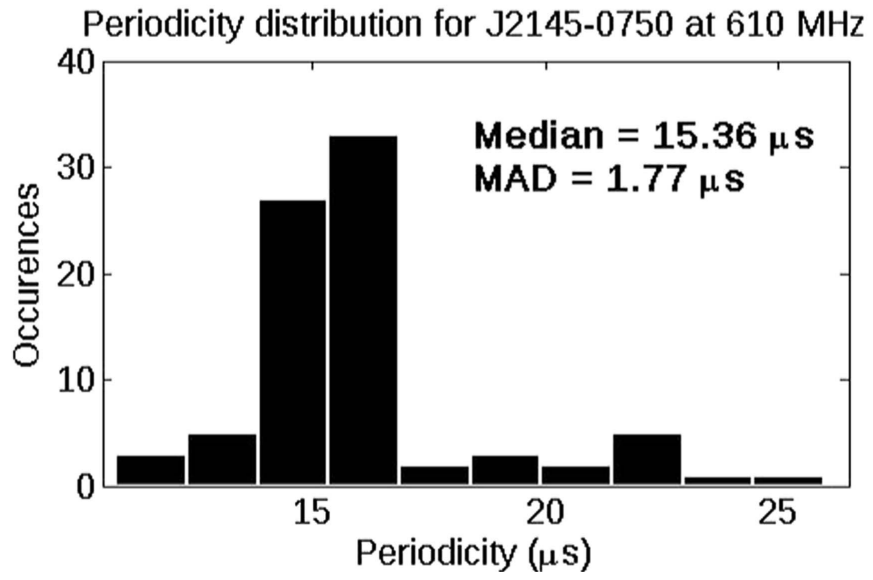

(d)

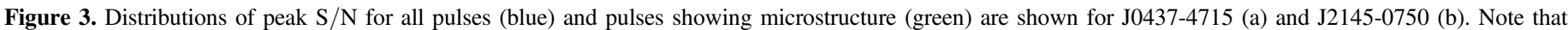

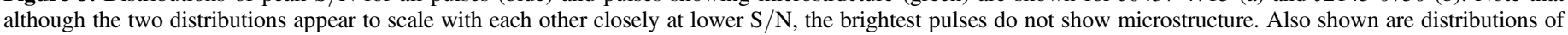

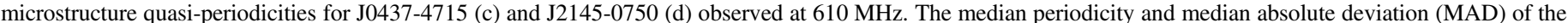

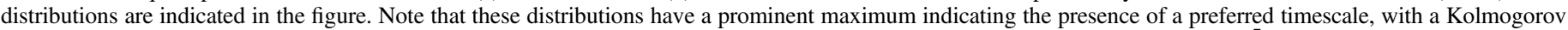
Smirnov null hypothesis (of the distribution having no preferred timescale, i.e., being a uniform distribution) probability of $\sim 10^{-7}$ in both cases.

Finally, we note that while we have presented here an analysis of the timescales of Stokes $I$ only, we were also able to measure the periodicities of Stokes $V$ and Stokes $L$ in cases where micropulses were highly polarized. While we did find that the periodicities in Stokes $I, V$, and $L$ were largely similar, the lower $\mathrm{S} / \mathrm{N}$ of the Stokes $V$ and $L$ profiles prevented a statistically accurate conclusion about their consistency. Additionally, the low $\mathrm{S} / \mathrm{N}$ of the Stokes $L$ profiles did not allow a close examination of the behavior of the PA across individual micropulses. Indeed, recent reports by Mitra et al. (2015) have suggested that the periodicities in these parameters are consistent with each other, leading them to argue that microstructure does not arise from single particle curvature radiation in vacuum.

However, the stability of the micropulse timescale-rotation period relationship down to millisecond periods does support a geometric origin of microstructure, wherein micropulses are formed by sweeping beams of radiation arranged longitudinally across the emission region (with constant angular widths $\sim 0^{\circ}$.18). We note that the reported inconsistency of the polarization signatures only rule out single particle curvature radiation as the origin of micropulse emission, but do not necessarily rule out a geometric origin. Additionally, a radial/ temporal origin of micropulse production is also a plausible alternative. In this model, micropulses arise from a radial modulation of the emission region, created either during the plasma production process or by modulation during propagation. It must be noted, however, that the modulation mechanism must be directly associated with the pulsar rotation period (for example, by means of rotation dependent electromagnetic fields; Cordes et al. 1990) to account for the strong dependence of periodicity with the rotation period. Clearly, future theoretical progress in this field coupled with observations of a larger number of pulsars are required to resolve these issues.

\section{SUMMARY}

In this Letter, we have presented the first report of detections and statistics of quasi-periodic microstructure emission in recycled MSPs. We find these micropulses to be often highly polarized, forming typically weak modulations on a broader subpulse envelope. By estimating microstructure periodicities from the single pulse ACFs, we report the first detections of "preferred" microstructure periodicity in MSPs. This result allows us to extend the microstructure timescale-rotation period relationship by more than an order of magnitude, and thus put constraints on the geometric sweeping and temporal modulation models of micropulse emission. While we cannot 


\section{Microstructure period against Rotation period}

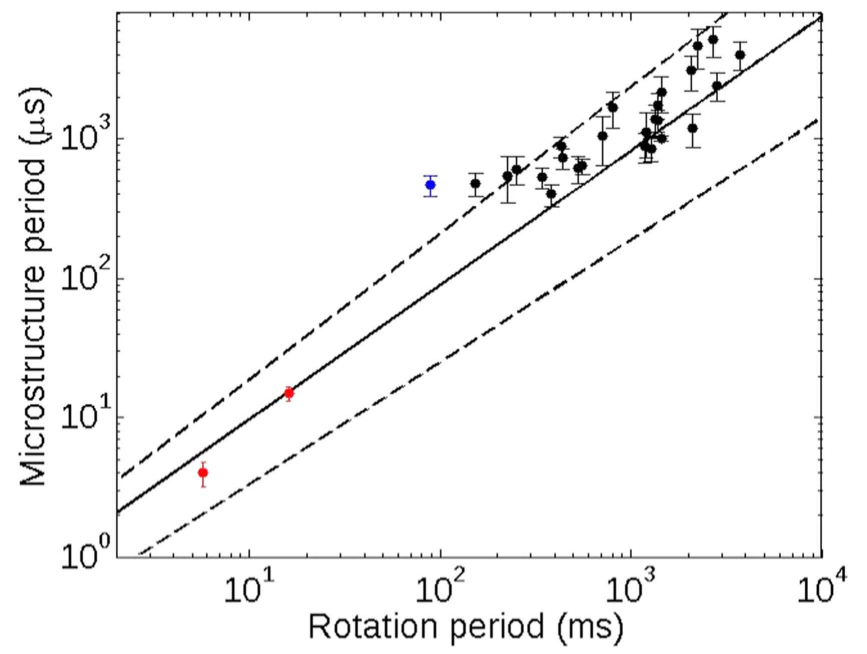

Figure 4. Microstructure periods of pulsars with known microstructure properties plotted against their rotation period. The red points are the ones added from this study, while the blue point corresponds to the reported microstructure periodicity for the Vela pulsar-the fastest rotating pulsar for which microstructure had been studied previously. The solid line corresponds to the best-fit power-law relationship between these quantities, while the dashed lines correspond to the $95 \%$ confidence interval on this relationship.

draw statistically significant conclusions about the detailed polarization signatures of micropulses from our data, future high-sensitivity observations at low frequencies are expected to resolve these aspects. In particular, the significantly larger bandwidths of the upgraded GMRT, which is currently being commissioned, is likely to allow an even larger-scale study of microstructure emission in MSPs, encompassing not only their full polarization properties but also their spectral evolution characteristics, explored using simultaneous dual-frequency observations.
We thank the staff of the GMRT who have made these observations possible. GMRT is run by the National Centre for Radio Astrophysics of the Tata Institute of Fundamental Research. We would like to thank R. T. Gangadhara for valuable discussions, as well as the anonymous referee for comments that helped significantly improve the paper. K.D. acknowledges support from a fellowship provided by the Kishore Vaigyanik Protsahan Yojana (KVPY) scheme of the Department of Science and Technology, Government of India. Partial support was provided by J. C. Bose Fellowship of Prof. Arnab Rai Choudhuri. This research has made use of NASA's Astrophysics Data System Bibliographic Services.

\section{REFERENCES}

Cordes, J. M. 1979, AuJPh, 32, 9

Cordes, J. M., \& Lazio, T. J. W. 2002, arXiv:astro-ph/0207156

Cordes, J. M., Weisberg, J. M., \& Hankins, T. H. 1990, AJ, 100, 1882

Craft, H. D., Comella, J. M., \& Drake, F. D. 1968, Natur, 218, 1122

De, K., \& Gupta, Y. 2016, ExA, 41, 67

Ferguson, D. C., \& Seiradakis, J. H. 1978, A\&A, 64, 27

Gupta, Y., Gothoskar, P., Joshi, B. C., et al. 2000, in ASP Conf. Ser. 202,

Pulsar Astronomy-2000 and Beyond, ed. M. Kramer, N. Wex, \&

N. Wielebinski (San Francisco, CA: ASP), 277

Hankins, T. H. 1972, ApJL, 177, L11

Hankins, T. H., Cordes, J. M., \& Rickett, B. J. 1976, BAAS, 8, 10

Jenet, F. A., Anderson, S. B., Kaspi, V. M., et al. 1998, ApJ, 498, 365

Jenet, F. A., Anderson, S. B., \& Prince, T. A. 2001, ApJ, 546, 394

Kramer, M., Johnston, S., \& van Straten, W. 2002, MNRAS, 334, 523

Kramer, M., Lange, C., Lorimer, D. R., et al. 1999, ApJ, 526, 957

Kramer, M., Xilouris, K. M., Lorimer, D. R., et al. 1998, ApJ, 501, 270

Lange, C., Kramer, M., Wielebinski, R., et al. 1998, A\&A, 332, 111

Liu, K., Bassa, C. G., Janssen, G. H., et al. 2016, MNRAS, 463, 3239

Manchester, R. N., Hobbs, G. B., Teoh, A., et al. 2005, AJ, 129, 1993

Mitra, D., Arjunwadkar, M., \& Rankin, J. M. 2015, ApJ, 806, 236

Navarro, J., Manchester, R. N., Sandhu, J. S., et al. 1997, ApJ, 486, 1019

Roy, J., Gupta, Y., Pen, Ue-Li, et al. 2010, ExA, 28, 25

Sallmen, S. M. 1998, PhD thesis, Univ. California

Stairs, I. H., Thorsett, S. E., \& Camilo, F. 1999, ApJS, 123, 627

Swarup, G., Ananthakrishnan, S., Subrahmanya, C. R., et al. 1997, in HighSensitivity Radio Astronomy, Proceedings of a Meeting Held at Jodrell Bank, University of Manchester, January 22-26, 1996, ed. D. Jackson, 217 